\title{
Prospective, Non-randomized, 36-Month Study of Second-Generation Trabecular Micro-Bypass Stents with Phacoemulsification in Eyes with Various Types of Glaucoma
}

Fritz H. Hengerer · Gerd U. Auffarth · Christoffer Riffel •

Ina Conrad-Hengerer

Received: September 28, 2018 / Published online: November 14, 2018

(C) The Author(s) 2018

\section{ABSTRACT}

Introduction: The aim of this study was to assess 36-month outcomes after cataract surgery and implantation of two second-generation trabecular micro-bypass stents (iStent inject, Glaukos Corporation, San Clemente, CA, USA) into eyes with predominantly primary open-angle glaucoma (POAG) or pseudoexfoliative glaucoma (PEX).

Methods: This prospective, non-randomized, consecutive cohort study included eyes with POAG $(n=60)$, PEX $(n=15)$, appositional narrow-angle $(n=4)$, pigmentary $(n=1)$, or neovascular (secondary) $(n=1)$ glaucoma and cataract requiring surgery. All eyes $(n=81)$ underwent $\mathrm{ab}$ interno iStent inject implantation following cataract surgery. Effectiveness endpoints through 36 months included intraocular pressure (IOP), number of medications, and proportion of eyes with $\geq 20 \%$ IOP reduction, IOP $\leq 18 \mathrm{mmHg}$, and $\mathrm{IOP} \leq 15 \mathrm{mmHg}$. Safety measures included corrected distance visual

Enhanced Digital Features To view enhanced digital features for this article go to https://doi.org/10.6084/ m9.figshare.7235723.

F. H. Hengerer $(\bowtie)$ · G. U. Auffarth · C. Riffel . I. Conrad-Hengerer

University Eye Hospital, University of Heidelberg,

Heidelberg, Germany

e-mail: f.hengerer@buergerhospital-ffm.de;

mail@augenarzt-trebur.de acuity (CDVA), adverse events, and secondary surgeries. Outcomes were evaluated for the overall cohort, and for the POAG and PEX subgroups.

Results: Preoperatively, $32.1 \%$ of eyes had undergone prior glaucoma surgery, $56 \%$ were on 3-4 medications, and 1 eye (1\%) was medication-free. At 36 months postoperatively, mean IOP reduced by $37 \%(14.3 \pm 1.7 \mathrm{mmHg}$ versus $22.6 \pm 6.2 \mathrm{mmHg}$ preoperatively), and mean medication burden decreased by $68 \%$ $(0.8 \pm 0.9$ versus $2.5 \pm 1.1$ medications preoperatively). IOP reduced by $\geq 20 \%$ in $78 \%$ of eyes; $100 \%$ of eyes reached $\mathrm{IOP} \leq 18 \mathrm{mmHg}$ and $71 \%$ reached $\leq 15 \mathrm{mmHg}$. Medication burden reduced considerably: 22 eyes (54\%) were medication-free compared to 1 eye $(1 \%)$ preoperatively; 1 eye $(2 \%)$ required $\geq 3$ medications compared to 45 eyes (56\%) preoperatively; and $92.7 \%$ of eyes required reduced medications postoperatively. From 3 through 36 months, mean IOP remained $\leq 15.0 \mathrm{mmHg}$, and mean number of medications remained $\leq 0.9$. Outcomes in the POAG and PEX subgroups included 33\% and 32\% lower IOP, and $68 \%$ and $64 \%$ fewer medications, respectively. iStent inject showed a favorable safety profile, including no intraoperative complications, minimal adverse events, and a stable CDVA.

Conclusion: This real-world cohort of eyes with various types of glaucoma and considerable disease burden exhibited durable and safe IOP and medication reductions through 36 months 
following iStent inject implantation with cataract surgery. Outcomes were similarly beneficial in eyes with POAG and PEX.

Funding: Article processing charges were funded by Glaukos Corporation (San Clemente, CA, USA).

Keywords: Cataract; Glaucoma; Intraocular pressure; iStent inject; Microinvasive glaucoma surgery (MIGS); PEX; Second-generation; Stent; Trabecular bypass

\section{INTRODUCTION}

Although filtering surgeries such as tube shunt implantation and trabeculectomy are the procedures most broadly utilized for glaucoma worldwide, the possibility of long-term complications remains a valid concern for both patients and physicians [1]. Since life expectancy and glaucoma prevalence are increasing in the US and globally [2, 3], and the cumulative risk of many complications increases over time, any given complication introduces its own years- or decades-long risk profile. For patients needing a dramatic reduction in intraocular pressure (IOP), these risks may be appropriate and necessary. However, for patients in earlier stages of the disease, and often even for patients with moderate and severe disease, a lower-risk trabecular microinvasive glaucoma surgery (MIGS) may offer sufficient and substantial reductions in IOP and medication burden while also maintaining consistent safety (and hence a desirable benefit-risk profile) over the long term [4].

Available MIGS treatment options have expanded greatly since the first MIGS device was introduced, the iStent ${ }^{\circledR}$ trabecular microbypass (Glaukos Corporation, San Clemente, CA, USA; FDA 2012; CE 2004). This stent is implanted ab interno with or without cataract surgery in accordance with the relevant country's approved indication. A significant body of evidence has shown meaningful long-term IOP and medication reductions after implanting one or multiple iStent devices, with excellent safety [5-22]. More recent years have seen the development and increasing surgical use of the second-generation iStent ${ }^{\circledR}$ inject trabecular micro-bypass (Glaukos; FDA 2018; CE 2010), which includes two trabecular stents implanted ab interno through the trabecular meshwork into Schlemm's canal in order to increase aqueous outflow and decrease IOP. A growing body of research has documented iStent inject performance and safety for mild to moderate OAG. The majority of these studies have evaluated stent implantation as a standalone procedure [23-27], while a smaller number of studies have assessed implantation combined with phacoemulsification [28-33]. In both settings, IOP and medication burden have shown clinically meaningful reductions and long-term durability, and the stent has presented favorable long-term safety.

The current report presents a single surgeon's experience of the 36 months following the implantation of two second-generation trabecular micro-bypass stents in eyes with various glaucoma diagnoses and a considerable preoperative medication and surgical burden. Safety and effectiveness outcomes are reported for the overall cohort and for the primary open-angle glaucoma (POAG) and pseudoexfoliative glaucoma (PEX) subgroups. As such, this report aims to provide practical, clinically relevant information for patients and surgeons who are deciding upon treatment for glaucoma.

\section{METHODS}

\section{Study Design}

This was a prospective, non-randomized, consecutive case series of eyes with various types of glaucoma and cataract that underwent implantation of two second-generation trabecular micro-bypass stents (Fig. 1) with cataract surgery. All surgeries were completed by a single surgeon (F.H.) over a 36-month period at an academic ophthalmology center in Heidelberg, Germany. Subjects were required to have glaucoma (including POAG, appositional narrowangle glaucoma [NAG, defined as a Shaffer grade of 2, with an open angle in the area of stent implantation], PEX, PG, or neovascular [secondary] glaucoma), and to be eligible for stent- 

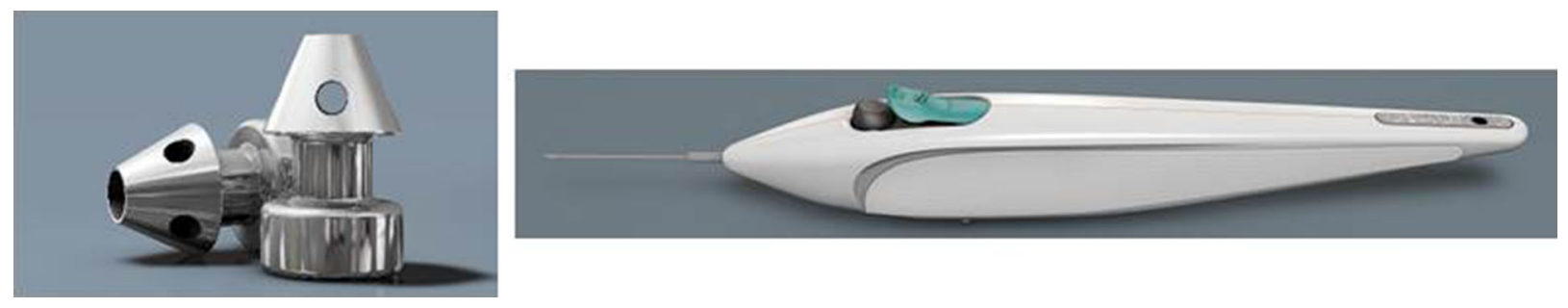

Fig. 1 iStent ${ }^{\circledR}$ inject stents and the iStent ${ }^{\circledR}$ inject trabecular micro-bypass stent system

cataract surgery. Additional inclusion criteria consisted of glaucoma progression despite sustained topical antiglaucomatous therapy. Patients were excluded if they had active intraocular inflammation, corneal opacities preventing gonioscopic viewing, angle closure, or any condition precluding safe concomitant cataract surgery. Additional exclusion criteria consisted of pregnancy, age under 18 years, and congenital glaucoma.

Preoperative and postoperative effectiveness evaluations included IOP (3 measurements per time point, measured by Goldmann applanation) and topical ocular hypotensive medications. Baseline IOP was determined by IOP readings at two or more preoperative visits. Safety analyses included CDVA, intraoperative complications, postoperative adverse events, and secondary surgical interventions. All procedures performed were in accordance with the ethical standards of the institutional and/or national research committee (the institutional review board [IRB] of the University of Heidelberg), and with the 1964 Helsinki Declaration and its later amendments or comparable ethical standards. Informed consent was obtained from all individual participants included in the study. Patients have already been followed for 36 months postoperatively, and follow-up is ongoing.

\section{Stent Description, Surgical Technique, and Perioperative Medication}

iStent inject implantation was completed following standard phacoemulsification and intraocular lens (IOL) implantation. As described previously [23-31], the iStent inject implantation technique may be summarized as follows: after pupillary miosis with a cholinergic agent (carbachol), the anterior chamber is filled with a medium-viscosity ocular viscosurgical device (OVD) and the surgeon then guides a single-use stainless steel injector through a temporal clear corneal incision in order to deliver two pre-loaded titanium stents $a b$ interno into the nasal Schlemm's canal approximately two clock-hours apart. Each heparin-coated $360 \mu \mathrm{m} \times 230 \mu \mathrm{m}$ stent has multiple lateral outlet lumens for fluid outflow and a symmetric design facilitating implantation in either the right or the left eye (Fig. 1). The stents are designed to decrease IOP by increasing trabecular outflow from the anterior chamber to Schlemm's canal. After surgery, patients received 1 week of topical antibiotic (ciprofloxacin; Floxal ${ }^{\circledR}$, Bausch \& Lomb, Berlin, Germany) and 4 weeks of topical anti-inflammatory medication (dexamethasone, Bausch \& Lomb, Berlin, Germany).

\section{Data Analyses}

Data analyses for the set of 81 eyes included mean IOP and number of medications used preoperatively and through 36 months postoperatively. Proportional analyses preoperatively and at 36 months included percentages of eyes with $\geq 20 \%$ IOP reduction, IOP $\leq 18 \mathrm{mmHg}$, and $\mathrm{IOP} \leq 15 \mathrm{mmHg}$; proportion of eyes on $3-4$ medications or $0-1$ medication; and proportion of eyes achieving a reduction in the number of medications at month 36 versus the preoperative regimen. Outcomes were analyzed for the overall cohort and for the POAG and PEX subgroups. In order to prevent inaccurate conclusions due to small sample size, subgroup analyses were performed at 12 months, when 
more patients had completed the follow-up (vs. at 36 months).

\section{RESULTS}

\section{Subject Accountability, Demographics, and Preoperative Parameters}

This cohort included a total of 81 eyes in 55 consecutive patients with POAG ( $n=60$ eyes), PEX ( $n=15$ eyes), appositional NAG $(n=4$ eyes), pigmentary glaucoma $(n=1$ eye), or neovascular (secondary) glaucoma ( $n=1$ eye) that underwent second-generation trabecular micro-bypass implantation in the setting of cataract surgery (Table 1). All subjects were Caucasian. At baseline, $32.1 \%$ of the eyes had undergone prior glaucoma surgery, $56 \%$ were on 3-4 preoperative medications, and 1 eye (1\%) was medication-free. Mean preoperative IOP was $22.6 \pm 6.2 \mathrm{mmHg}$ and mean medication burden was $2.5 \pm 1.1$ medications.

In the subgroup of eyes with POAG $(n=60)$, preoperative parameters were generally similar to the overall cohort, with a mean preoperative IOP of $21.92 \mathrm{mmHg}$ and a mean medication burden of 2.35 medications. In comparison, eyes with PEX $(n=15)$ had a higher mean preoperative IOP (23.3 $\mathrm{mmHg}$ ) and a higher mean medication burden (2.8 medications).

\section{Intraocular Pressure and Medication Use}

In all eyes that were followed-up for 36 months $(n=41)$, mean IOP decreased to $14.3 \pm 1.7 \mathrm{mmHg}$ versus $22.6 \pm 6.2 \mathrm{mmHg}$ preoperatively (37\% reduction; Fig. 2$)$, and

Table 1 Demographics and preoperative characteristics $(n=81$ eyes of 55 patients)

\begin{tabular}{ll}
\hline Mean $\mathbf{\pm}$ SD & iStent inject + phaco $\mathbf{8 1}$ eyes/55 patients) \\
\hline Gender & 45 males/36 females \\
Age & $72.7 \pm 7.5 \mathrm{yrs}$ \\
(Range) & $(54-86)$ \\
Race & $100 \%$ Caucasian \\
C:D ratio & $0.7 \pm 0.1$ \\
VF MD (dB) & $-8.2 \pm 6.6 \mathrm{~dB}$ \\
Type of glaucoma, $n$ (\%) & \\
POAG & $60(74 \%)$ \\
PEX & $15(19 \%)$ \\
Appositional narrow-angle & $4(5 \%)$ \\
Pigmentary & $1(1 \%)$ \\
Neovascular (secondary to CRVO) & $1(1 \%)$ \\
Preoperative medicated IOP & $22.6 \pm 6.2 \mathrm{mmHg}$ \\
Mean \# medications & $2.5 \pm 1.1$ \\
Eyes with prior glaucoma surgery, $n(\%)$ & $26(32.1 \%)$ \\
Eyes on 3-4 preoperative medications $(\%)$ & $56 \%$ \\
\hline
\end{tabular}

$S D$ standard deviation, $C: D$ cup-to-disc, $V F M D$ visual field mean deviation, $C R V O$ central retinal vein occlusion

${ }^{a}$ Eyes had an open-angle configuration in the area of iStent inject implantation 


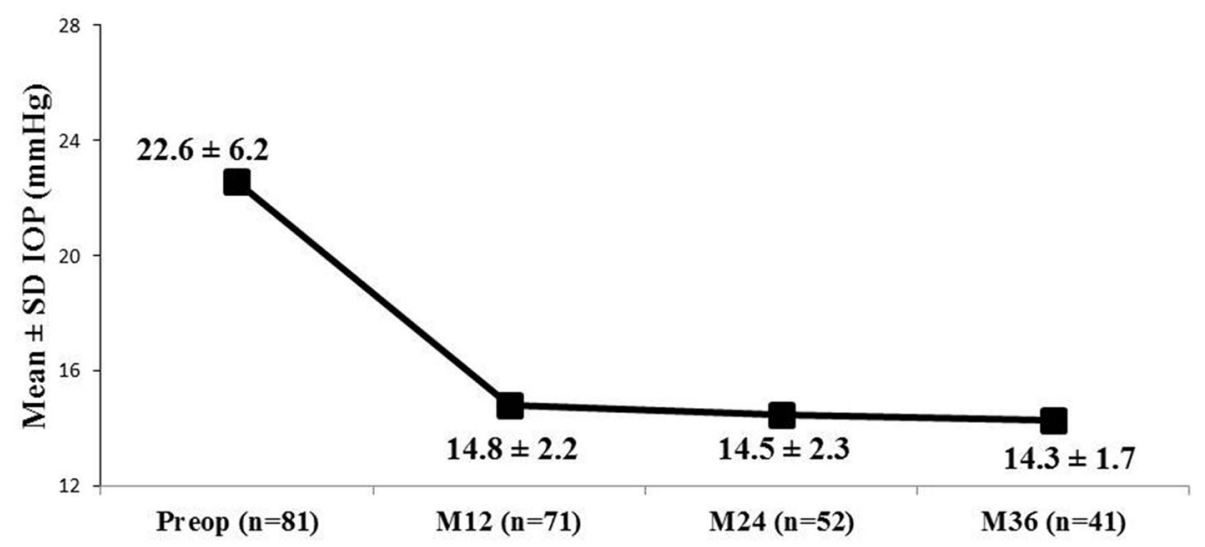

Fig. 2 Mean intraocular pressure (IOP) through 36 months; eyes with data available for each visit are included. IOP intraocular pressure, $S D$ standard deviation, $M$ month, Preop preoperative

mean medication burden reduced to $0.8 \pm 0.9$ versus $2.5 \pm 1.1$ medications preoperatively (68\% reduction; Fig. 3). A $\geq 20 \%$ IOP reduction was achieved in $78 \%$ of eyes, with $100 \%$ of eyes reaching $\mathrm{IOP} \leq 18 \mathrm{mmHg}$ and $71 \%$ reaching $\leq 15 \mathrm{mmHg} ;$ Fig. 4). Medication burden also decreased: at 36 months, $54 \%$ of eyes $(n=22)$ were medication-free compared to $1 \%$ $(n=1)$ preoperatively; conversely, $2 \%$ of eyes $(n=1)$ were on $\geq 3$ medications compared to

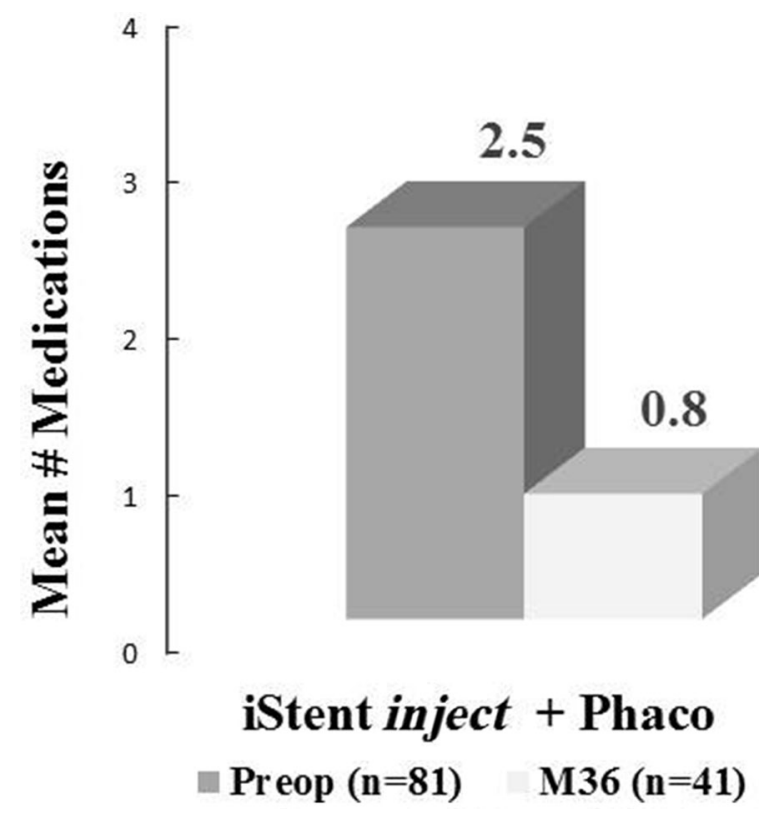

Fig. 3 Mean medication burden at 36 months versus preoperative burden; eyes with data available for each visit are included. $M$ month, Preop preoperative

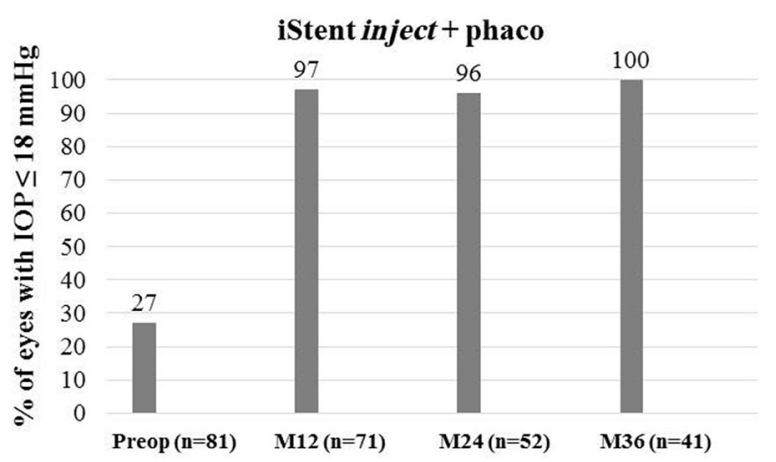

Fig. 4 Proportional analysis of IOP $\leq 18 \mathrm{mmHg}$ through 36 months; eyes with data available for each visit are included. IOP intraocular pressure, $M$ month, Preop preoperative

$56 \%$ of eyes $(n=45)$ preoperatively (Fig. 5). At 36 months, $92.7 \%$ of the eyes had decreased the number of medications from their preoperative regimens. At all postoperative visits from 3 months through 36 months, mean IOP was maintained at $\leq 15.0 \mathrm{mmHg}$ and mean medication burden was limited to 0.9 or fewer medications.

In POAG eyes, at the 12-month follow-up $(n=55)$, mean IOP had decreased by $33 \%$ to $14.75 \mathrm{mmHg}$ vs. 21.92 preoperatively and medication burden had decreased by $68 \%$ to 0.76 versus 2.35 medications preoperatively. In PEX eyes, at the 12-month follow-up $(n=10)$, mean IOP had decreased by $32 \%$ to $15.9 \mathrm{mmHg}$ versus $23.3 \mathrm{mmHg}$ preoperatively and 


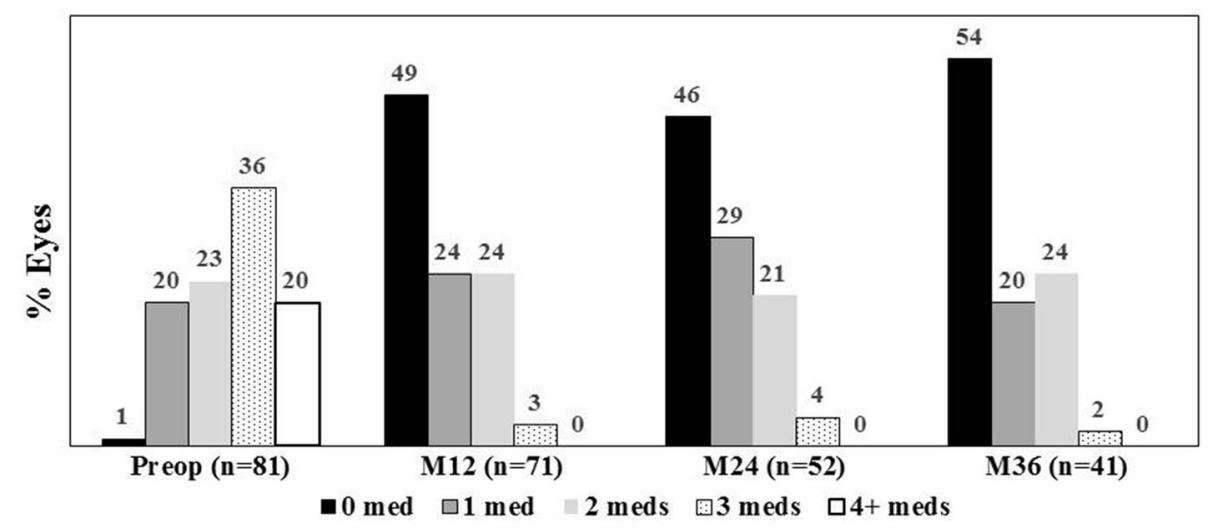

Fig. 5 Proportional analysis of medication use through 36 months; eyes with data available for each visit are included. $M$ month, Preop preoperative, Med medication

medication burden had decreased by $64 \%$ to 1.0 versus 2.8 medications preoperatively.

\section{Safety Assessment}

All patients underwent uneventful implantation of two iStent inject stents following standard phacoemulsification cataract surgery. No intraoperative complications occurred. Two eyes developed corneal edema during the first day after cataract removal; both cases resolved completely with standard topical therapy by 5 days postoperatively. No additional adverse events occurred in any eye during the remainder of the follow-up period. Postoperative CDVA was maintained at $20 / 40$ or better in all but one eye $(26 / 27$ or $96 \%$; 12 -month data); the one remaining eye had a CDVA of 20/50. Three eyes underwent additional glaucoma procedures during the follow-up period due to increased IOP or disease progression unrelated to stent implantation. These interventions included cyclophotocoagulation (CPC) in 1 eye at month 1, CPC in 1 eye at month 6 , and XEN implantation at month 6 .

\section{DISCUSSION}

The current report provides data on outcomes of iStent inject implantation combined with cataract surgery in a patient population with a sizeable preoperative medication burden and surgical history in a real-life clinical setting, making the data particularly meaningful for clinicians evaluating treatment options for their glaucoma patient population.

In these 81 eyes with POAG, PEX, appositional NAG, PG, or neovascular (secondary) glaucoma, substantial reductions in both IOP and medication use were observed for 36 months postoperatively following the implantation of two second-generation trabecular micro-bypass stents with cataract surgery. These favorable results are consistent with those of numerous previous studies of first-generation trabecular micro-bypass stent implantation in conjunction with cataract surgery for mild to moderate glaucoma [5-16]. The results also corroborate previous reports of IOP and medication reduction after implantation of two iStent devices in a standalone procedure [17-22]. In addition, the findings support prior research on second-generation iStent inject implantation either with or without cataract surgery in subjects with predominantly mild to moderate levels of IOP elevation or medication burden [23-33].

In the overall cohort, preoperative disease burden (as indicated by number of medications and history of prior glaucoma surgery) was relatively substantial. Over half of the eyes were being treated with 3-4 medications preoperatively, nearly one-third of the eyes had undergone prior glaucoma surgery (or surgeries), and only one eye was medication-free. In such previously operated eyes, remodeling and fibrosis 
of the trabecular meshwork and juxtacanalicular tissue have been shown to occur, thereby producing outflow resistance and contributing to glaucoma [34, 35]. The difficulty involved in achieving favorable outcomes in such eyes is widely acknowledged $[1,34]$, and is thought to depend on the patency of each eye's outflow network [35]. Our study showed that there were significant IOP and medication reductions in previously operated eyes, suggesting that trabecular micro-bypass stents could potentially circumvent the diseased portions of the outflow pathway to restore normal aqueous dynamics in the remaining healthy parts.

Given that stent implantation was completed in conjunction with cataract surgery, it is important to acknowledge the widely known impact that cataract surgery alone can have in reducing IOP [36-38]. Post-phacoemulsification IOP reduction is expected to be modest: less than $2 \mathrm{mmHg}$ on average [36, 37], or a reduction versus baseline of $16.5 \%$ three years after cataract extraction, as shown in the Ocular Hypertension Treatment Study (OHTS) [38]. Less dramatic IOP reductions are expected in the setting of lower baseline IOP (e.g., IOP $\leq 20 \mathrm{mmHg}$ ) $[39,40]$. In the present cohort, IOP decreased by over $8 \mathrm{mmHg}$, a reduction of $37 \%$; this reduction is approximately two to four times greater than the reduction expected with cataract surgery alone. This is consistent with clinical studies showing significantly greater reductions in IOP and medication use in patients with iStent and iStent inject implantation plus cataract surgery compared to cataract surgery alone [2-4, 31, 41-43]. It also aligns with the work of Fernandez-Barrientos et al. [2], who demonstrated significant increases in trabecular outflow facility at 12 months in eyes implanted with iStent in combination with cataract surgery compared to eyes with cataract surgery alone.

Safety parameters for iStent inject were consistently favorable throughout the follow-up period, with no sign of the long-term sequelae known to occur with traditional filtering surgeries or more recently developed procedures involving the subconjunctival space. Importantly, stent implantation did not preclude the visual improvement expected with cataract surgery, as evidenced by favorable and stable postoperative CDVA. Only two adverse events occurred (both consisting of corneal edema in the immediate postoperative period), and these cases resolved without sequelae within 5 days. Notably, there were no reports of hypotony, hypotony maculopathy, choroidal hemorrhage, endophthalmitis, PAS, or stent occlusion.

With regards to the POAG and PEX subgroups, preoperative characteristics were generally consistent with what has been reported in the literature. In particular, eyes with PEX had slightly higher preoperative IOP and medication burden than eyes with POAG, consistent with the more aggressive and treatment-recalcitrant nature of this glaucoma type [44]. Postoperatively, the outcomes in PEX eyes were similarly favorable to those in POAG eyes, thereby corroborating prior studies showing iStent technology to be a highly suitable and effective treatment option in PEX eyes [12, 14].

We acknowledge several limitations of this unmasked, single-arm study. Subjects comprised a consecutive series of patients in the surgeon's clinical practice, and thus were not required to meet narrow inclusion criteria for enrollment. Subjects also did not complete preoperative and postoperative medication washouts, since these are not usually part of standard clinical practice and could potentially cause harm to subjects who have moderate to advanced glaucoma. As is the case in most realworld clinical studies, there was no formal control group with which to compare the treatment group; however, we considered preoperative IOP and number of medications to be valid comparators given that they are numeric measures. Potential additional confounders include the absence of diurnal IOP measurements, regression to the mean, and the inability to separate the IOP effects of cataract extraction and stent implantation. In order to avoid misleading conclusions due to the limited number of eyes with complete follow-up through 36 months, subgroup analyses for PEX and POAG were performed at 12 months instead. Future reports based on this dataset could include analyses through 36 months or longer 
for these subgroups as well as for the overall cohort. Other directions for future work could include stratifying outcomes by type of prior glaucoma surgery and/or number of preoperative medications; examining long-term RNFL data (via OCT measures) as a structural correlate for disease control; and comparing postoperative 3- or 6-month refractive data with expected preoperative values.

\section{CONCLUSIONS}

In conclusion, this series demonstrated substantial reductions in both IOP and medication burden through 36 months postoperatively following the implantation of two second-generation trabecular micro-bypass stents with cataract surgery, along with favorable safety. Importantly, these outcomes were observed in a clinically heterogeneous patient population with a considerable preoperative medication burden and prevalence of prior glaucoma surgery. This diversity and real-world setting could make the data more representative of real-life clinical populations than many product registration trials. Thus, the study provides a useful reference for both clinicians and patients who are evaluating their glaucoma treatment options.

\section{ACKNOWLEDGEMENTS}

We thank the participants in the study.

Funding. No sponsorship was received for completing this study. Article processing charges were funded by Glaukos Corporation (San Clemente, CA, USA). All authors had full access to all of the data in this study and take complete responsibility for the integrity of the data and accuracy of the data analysis.

Editorial Assistance. Editorial assistance in the preparation of this article was provided by Dr. Dana M. Hornbeak, MD, MPH of Glaukos Corporation. This assistance was funded by Glaukos Corporation (San Clemente, CA, USA).
Authorship. All named authors meet the International Committee of Medical Journal Editors (ICMJE) criteria for authorship for this article, take responsibility for the integrity of the work as a whole, and have given their approval for this version to be published.

Prior Presentation. Presented in part at the 2018 annual meetings of the American Society of Cataract and Refractive Surgery (ASCRS), the European Glaucoma Society (EGS), and the European Society of Cataract and Refractive Surgeons (ESCRS).

Disclosures. Christoffer Riffel declares that he has no conflicts of interest. Fritz H. Hengerer has received travel support, research grants, and lecture fees from Glaukos Corporation. Gerd U. Auffarth has received travel support, research grants, and lecture fees from Glaukos Corporation. Ina Conrad-Hengerer has received travel support, research grants, and lecture fees from Glaukos Corporation.

Compliance with Ethics Guidelines. All procedures performed were in accordance with the ethical standards of the institutional and/or national research committee (the Institutional Review Board (IRB) of the University of Heidelberg), and with the 1964 Helsinki Declaration and its later amendments or comparable ethical standards. Informed consent was obtained from all individual participants included in the study.

Data Availability. The datasets generated during and/or analyzed during the current study are available from the corresponding author on reasonable request.

Open Access. This article is distributed under the terms of the Creative Commons Attribution-NonCommercial 4.0 International License (http://creativecommons.org/licenses/ by-nc/4.0/), which permits any noncommercial use, distribution, and reproduction in any medium, provided you give appropriate credit to the original author(s) and the source, provide a link to the Creative Commons license, and indicate if changes were made. 


\section{REFERENCES}

1. Gedde SJ, Herndon LW, Brandt JD, Budenz DL, Feuer WJ, Schiffman JC; Tube Versus Trabeculectomy Study Group. Postoperative complications in the Tube Versus Trabeculectomy (TVT) study during 5 years of follow-up. Am J Ophthalmol 2012;153:804-814.

2. Tham YC, Li X, Wong TY, Quigley HA, Aung T, Cheng CY. Global prevalence of glaucoma and projections of glaucoma burden through 2040: a systematic review and meta-analysis. Ophthalmology. 2014;121:2081-90.

3. Quigley HA. Glaucoma. Lancet. 2011;377:1367-77.

4. Pillunat LE, Erb C, Jünemann AG, Kimmich F. Micro-invasive glaucoma surgery (MIGS): a review of surgical procedures using stents. Clin Ophthalmol. 2017;11:1583-600.

5. Samuelson TW, Katz LJ, Wells JM, Duh YJ, Giamporcaro JE. Randomized evaluation of the trabecular micro-bypass stent with phacoemulsification in patients with glaucoma and cataract. Ophthalmology. 2011;118:459-67.

6. Craven ER, Katz LJ, Wells JM, Giamporcaro JE. Cataract surgery with trabecular micro-bypass stent implantation in patients with mild-to-moderate open-angle glaucoma and cataract: two-year followup. J Cataract Refract Surg. 2012;38:1339-45.

7. Fea AM, Consolandi G, Zola M, et al. Micro-bypass implantation for primary open-angle glaucoma combined with phacoemulsification: 4-year followup. J Ophthalmol. 2015;795357. https://doi.org/10. $1155 / 2015 / 795357$

8. Arriola-Villalobos P, Martinez-de-la-Casa J, DiazValle D, Fernández-Pérez C, García-Sánchez J, García-Feijoó J. Combined iStent trabecular micro-bypass stent implantation and phacoemulsification for coexistent open-angle glaucoma and cataract: a long-term study. Br J Ophthalmol. 2012;96:645-9.

9. Belovay GW, Naqi A, Chan BJ, Rateb M, Ahmed II. Using multiple trabecular micro-bypass stents in cataract patients to treat open-angle glaucoma. J Cataract Refract Surg. 2012;38(11):1911-7.

10. Gallardo MJ, Supnet RA, Giamporcaro JE, Hornbeak DM. Outcomes of combined trabecular micro-bypass and phacoemulsification in a predominantly Hispanic patient population. Clin Ophthalmol. 2016;10:1931-7.

11. Ferguson TJ, Berdahl JP, Schweitzer JA, Sudhagoni RG. Clinical evaluation of a trabecular micro-bypass stent with phacoemulsification in patients with open-angle glaucoma and cataract. Clin Ophthalmol. 2016;10:1767-73.

12. Ferguson TJ, Swan R, Ibach M, Schweitzer J, Sudhagoni R, Berdahl JP. Trabecular microbypass stent implantation with cataract extraction in pseudoexfoliation glaucoma. J Cataract Refract Surg. 2017;43(5):622-6.

13. Ferguson T, Swan R, Ibach M, Schweitzer J, Sudhagoni R, Berdahl JP. Evaluation of a trabecular microbypass stent with cataract extraction in severe primary open-angle glaucoma. J Glaucoma. 2018;27(1):71-6.

14. Neuhann TH. Trabecular micro-bypass stent implantation during small-incision cataract surgery for open-angle glaucoma or ocular hypertension: long-term results. J Cataract Refract Surg. $2015 ; 41: 2664-71$.

15. Buffet J, Brasnu E, Baudouin C, Labbé A. Efficacy of two trabecular micro-bypass stents during phacoemulsification for mild to advanced primary open-angle glaucoma controlled with topical hypotensive medications. J Glaucoma. 2017;26(12):1149-54.

16. Brown RH, Gibson Z, Zhong L, Lynch MG, Intraocular pressure reduction after cataract surgery with implantation of a trabecular microbypass device. J Cataract Refract Surg. 2015;41(6):1318-9.

17. Donnenfeld ED, Solomon KD, Voskanyan L, et al. A prospective 3-year follow-up trial of implantation of two trabecular microbypass stents in open-angle glaucoma. Clin Ophthalmol. 2015;9:2057-65.

18. Katz LJ, Erb C, Guillamet AC, Fea AM, Voskanyan L, Giamporcaro JE, Hornbeak DM. Long-term titrated IOP control with one, two, or three trabecular micro-bypass stents in open-angle glaucoma subjects on topical hypotensive medication: 42-month outcomes. Clin Ophthalmol. 2018;12:255-62.

19. Ahmed II, Katz LJ, Chang DF, Donnenfeld ED, Solomon KD, Voskanyan L, Samuelson TW. Prospective evaluation of microinvasive glaucoma surgery with trabecular microbypass stents and prostaglandin in open-angle glaucoma. J Cataract Refract Surg. 2014;40:1295-300.

20. Vold SD, Voskanyan L, Tetz M, et al. Newly diagnosed primary open-angle glaucoma randomized to 2 trabecular bypass stents or prostaglandin: outcomes through 36 months. Ophthalmol Ther. 2016;5(2):161-72.

21. Ferguson T, Berdahl J, Schweitzer J, Sudhagoni R. Evaluation of a trabecular micro-bypass stent in pseudophakic patients with open-angle glaucoma. J Glaucoma. 2016;25:896-900. 
22. Chang DF, Donnenfeld ED, Katz LJ, et al. Efficacy of two trabecular micro-bypass stents combined with topical travoprost in open-angle glaucoma not controlled on two preoperative medications: 3-year follow-up. Clinical Ophthalmology. 2017;11:523-8.

23. Fea AM, Belda JI, Rekas M, Junemann A, Chang L, Pablo L, Voskanyan L, Katz LJ. Prospective unmasked randomized evaluation of the iStent inject $^{\circledR}$ versus two ocular hypotensive agents in patients with primary open-angle glaucoma. Clinical Ophthalmology. 2014;8:875-82.

24. Klamann MKJ, Gonnermann J, Pahlitzsch M, et al. iStent inject in phakic open angle glaucoma. Graefes Arch Clin Exp Ophthalmol. 2015;253:941-7.

25. Lindstrom R, Lewis R, Hornbeak H, Voskanyan L, Giamporcaro JE, Hovanesian J, Sarkisian S. Outcomes following implantation of two second-generation trabecular micro-bypass stents in patients with open-angle glaucoma on one medication: 18-month follow-up. Adv Ther. 2016;33:2082-90.

26. Voskanyan L, Garcia-Feijoo J, Belda J, Fea A, Jünemann A, Baudouin C; Synergy Study Group. Prospective, unmasked evaluation of the iStent Inject system for open-angle glaucoma: synergy trial. Adv Ther 2014;31(2):189-201.

27. Berdahl J, Voskanyan L, Myers JS, et al. Implantation of two second-generation trabecular microbypass stents and topical travoprost in open-angle glaucoma not controlled on two preoperative medications: 18-month follow-up. Clin Exp Ophthalmol. 2017;45(8):797-802.

28. Arriola-Villalobos P, Martínez-de-la-Casa JM, DíazValle D, García-Vidal SE, Fernández-Pérez C, GarcíaSánchez J, García-Feijoó J. Mid-term evaluation of the new Glaukos iStent with phacoemulsification in coexistent open-angle glaucoma or ocular hypertension and cataract. $\mathrm{Br} \mathrm{J}$ Ophthalmol. 2013;97:1250-5.

29. Arriola-Villalobos P, Martinez-de-la-Casa J, DiazValle, Morales-Fernandez L, Fernandez-Perez C, Garcia-Feijoo J. Glaukos iStent inject trabecular micro-bypass implantation associated with cataract surgery in patients with co-existing cataract and open-angle glaucoma or ocular hypertension: a long-term study. J Ophthalmol. 2016;1056573. https://doi.org/10.1155/2016/1056573.

30. Gonnermann J, Bertelmann E, Pahlitzsch M, MaierWenzel AB, Torun N, Klamann MK. Contralateral eye comparison study in MICS and MIGS: Trabectome $^{\circledR}$ vs. iStent inject ${ }^{\circledR}$. Graefes Arch Clin Exp Ophthalmol 2017;255(2):359-365.
31. Macher T, Häberle $H$, Wächter J, Thannhäuser C, Aurich H, Pham DT. Trabecular microbypass stents as minimally invasive approach after conventional glaucoma filtration surgery. J Cataract Refract Surg. 2018;44(1):50-5.

32. Clement C. Audit of Australian outcomes with iStent Inject combined with cataract surgery. Presented at: Annual Meeting of the Asia-Pacific Glaucoma Congress; 13-15 Apr 2018; Busan, Korea.

33. Harasymowycz P. Single surgeon evaluation of second-generation trabecular micro-bypass stents in patients with mild to severe glaucoma. Presented at: Annual Meeting of the American Society of Cataract and Refractive Surgeons; 13-17 April 2018; Washington, DC, USA.

34. Tektas OY, Lutjen-Drecoll E. Structural changes of the trabecular meshwork in different kinds of glaucoma. Exp Eye Res. 2009;88:769-75.

35. Craven ER. Trabecular micro-bypass shunt (iStent $\left.{ }^{\circledR}\right)$ : basic science, clinical, and future. Middle East Afr J Ophthalmol. 2015;22(1):30-37.

36. Vizzeri G, Weinreb RN. Cataract surgery and glaucoma. Curr Opin Ophthalmol. 2010;21(1):20-4.

37. Friedman DS, Jampel HD, Lubomski LH, et al. Surgical strategies for coexisting glaucoma and cataract: an evidence-based update. Ophthalmology. 2002;109:1902-13.

38. Mansberger SL, Gordon MO, Jampel $\mathrm{H}$, et al. Reduction in intraocular pressure after cataract extraction: the ocular hypertension treatment study. Ophthalmology. 2012;119:1826-31.

39. Shingleton BJ, Gamell LS, O’Donoghue MW, Baylus SL, King R. Long-term changes in intraocular pressure after clear corneal phacoemulsification: normal patients versus glaucoma suspect and glaucoma patients. J Cataract Refract Surg. 1999;25(7):885-90.

40. Poley BJ, Lindstrom RL, Samuelson TW. Long-term effects of phacoemulsification with intraocular lens implantation in normotensive and ocular hypertensive eyes. J Cataract Refract Surg. 2008;34(5):735-42.

41. Samuelson TW. Prospective, randomized, multicenter clinical investigation of the Glaukos iStent inject. Presented at: Annual Meeting of the American Society of Cataract and Refractive Surgeons; 13-17 April 2018; Washington, DC, USA.

42. Clement C. Audit of Australian outcomes with iStent inject combined with cataract surgery. Presented at: Annual Meeting of the Asia-Pacific Glaucoma Congress; 13-15 April 2018; Busan, Korea. 
43. Harasymowycz P. Single surgeon evaluation of second-generation trabecular micro-bypass stents in patients with mild to severe glaucoma. Presented at: Annual Meeting of the American Society of Cataract and Refractive Surgeons; 13-17 April 2018; Washington, DC, USA.
44. Topouzis F, Harris A, Wilson MR, Koskosas A, Founti P, Yu F, Anastasopoulos E, Pappas T, Coleman AL. Increased likelihood of glaucoma at the same screening intraocular pressure in subjects with pseudoexfoliation: the Thessaloniki Eye Study. Am J Ophthalmol. 2009;148:606-13. 